\title{
Periodontal Disease as a Risk Factor for Cardiovascular Disease - A Questionnaire Survey among Physicians
}

\author{
Varun Arya ${ }^{1}$, Ramya Shenoy Kudpi ${ }^{2}$, Nikita Rungta ${ }^{3}$
}

\begin{abstract}
:
Introduction: Periodontal infections have been documented to influence coronary heart diseases such as angina, myocardial infarction, atherosclerosis and other systemic conditions like stroke, diabetes mellitus and preterm low birth weight babies. Over the last two decades, several studies have reported associations between periodontitis and cardiovascular disease (CVD). The association is now a part of the dental school curriculum and periodontology. However, in contrast, this important aspect is missing in the medical school curriculum and not many physicians are aware of the role periodontal disease plays in CVD.
\end{abstract}

Aim: To assess the knowledge and practices of physicians of Mangalore City, South India, on periodontal disease as a risk factor in Cardiovascular Diseases (CVD).

Methodology: A questionnaire survey was carried out to assess physicians' knowledge and practices in the city of Mangalore, South India. The physicians were divided into four groups as follows: Group I Physicians associated with medical colleges; Group II - Sole private practitioners; Group III - Physicians associated with medical colleges and having their private practice; Group IV - Post-graduate students. A Chi-square test was applied to test the difference between knowledge and practices among the groups.

Results: Out of a total of 106 physicians, 56 (52.3\%) participated in the survey. Periodontal disease was considered to be a risk factor in CVD by $87.5 \%(n=47)$ respondents and $96.4 \%(n=54)$ respondents stated that bleeding/enlargement of the gingivae was a sign of periodontal infection. Only $40 \%(n=23)$ of the respondents asked about oral problems during general check-up.

Conclusions: The knowledge regarding the periodontal disease as a risk factor in CVD was high, however, the application of the knowledge in routine medical check-ups was observed to be less.

Keywords: physicians', knowledge, periodontal disease, cardiovascular disease (CVD), periodontitis

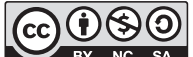

DOI: https://doi.org/l0.3329/jom.v22il.5/384

Copyright: (c) 2020 Hossain MM et al. This is an open access article published under the Creative Commons Attribution-NonCommercial-NoDerivatives 4.0 International License, which permits use, distribution and reproduction in any medium, provided the original work is properly cited, is not changed in any way and it is not used for commercial purposes.

Received: 26 June, 2020

Accepted: 28 November, 2020

\section{Introduction:}

WHO statistics indicate that more people die annually around the globe from cardiovascular diseases (CVD) as compared to any other diseases. It was estimated that around 17.9

1. Oral and Maxillofacial Surgeon, Private Practice, Wood and Myers Oral Surgery, 207 S $32^{\text {nd }}$ Street, Camp Hill, Pennsylvania, USA 17011

2. Associate Professor, Department of Public Health Dentistry, Manipal College of Dental Sciences, Mangalore, Manipal Academy of Higher Education (MAHE), Manipal, India

3. Research Assistant, Manipal College of Dental Sciences, Mangalore, Manipal Academy of Higher Education (MAHE), Manipal, India

Corresponding author: Dr. Ramya Shenoy Kudpi, Associate Professor, Department of Public Health Dentistry, Manipal College of Dental Sciences, Mangalore, Manipal Academy of Higher Education (MAHE), Manipal, India. E-mail: ramya.shenoy@, manipal.edu million people died from CVDs in 2016, representing 31\% of all the global deaths. Of these deaths, $85 \%$ were due to heart attack and stroke. ${ }^{1}$ Current evidence has shed light on the relationship between systemic health including CVDs and oral health. It has been reported that inflammation of gums and loss of alveolar bone (periodontitis) can be one of the risk factors associated with CVDs. Over the last 20 years, several studies have reported epidemiological associations between periodontitis and cardiovascular disease. ${ }^{2}$ Patients with periodontitis are at increased risk for thickening of the walls of major coronary arteries. Multiple species of periodontal pathogens have been observed in more than half of the atheromas obtained from humans during endarterectomy. ${ }^{3}$

Periodontitis is a chronic inflammatory disease, initiated by a bacterial biofilm called dental plaque, which affects the 
periodontal ligaments and bone surrounding the teeth. Several studies have suggested the existence of a bidirectional link between periodontal health and CVDs. People with diabetes are more likely to suffer from periodontitis than people without this syndrome. Analogously, it is now evident that cardiac disorders are worsened by periodontitis, both in humans and experimentally. Therefore, it is very crucial that preventing periodontitis has an impact on the onset or progression of CVD and diabetes. ${ }^{4}$ It has also been established that in response to LPS (lipopolysaccharide) /endotoxin from the bacterial micro-organisms, endogenous host products are produced that can activate the host immune response, releasing cytokines, TNF-alpha, prostaglandins, and matrix metalloproteinases. The resulting bacteremia and endotoxemia can initiate a strong systemic antibody response and potentially can affect the systemic health of an individual. Contemporarily, it is well established that periodontal bacteria can invade the systemic circulation also. ${ }^{5}$ Oral pathogens and inflammatory mediators (IL-1 $\beta$, TNF- $\alpha$ ) from periodontal lesions intermittently reach the bloodstream inducing chronic low-level bacteremia and systemic inflammatory reactants (C-reactive protein, systemic antibodies), all of which may represent a pathogenetic link between periodontal disease and heart disease (CVD). ${ }^{6}$

Integration of periodontal therapy such as prophylaxis, scaling and root planning along with antibiotic prophylaxis into medical management of systemic disease may have a positive systemic health outcome. This crucial relationship is important for both dental health care providers and those in the primary care and cardiovascular setting to understand and reduce the morbidity associated with CVD. It may also contribute to saving the financial burden on health care systems and the affected families associated with these systemic diseases. ${ }^{7}$ Therefore, this questionnaire survey was conducted with the aim of assessing the general physician's knowledge and practices regarding periodontal disease as a risk factor for cardiovascular diseases.

\section{Materials and Methods:}

Permission from the Head of the Institute was obtained to conduct the survey. The present questionnaire survey was conducted within Mangalore city, South India. The lists of physicians/medical practitioners were obtained from the office of the Indian Medical Association, Mangalore branch, Karnataka. Informed consent was obtained from each subject before them filling the questionnaire.

\section{Inclusion Criteria}

Physicians/practitioners who have completed or are pursuing MD in General Medicine (Doctors of Medicine), recognized by the Medical Council of India, were included for the survey. Based on the inclusion criteria, a total of 106 subjects (physicians) were selected to be included in the survey and were divided into four groups as follows:

Group I - Physicians in medical colleges

Group II - Sole Private Practitioners

Group III - Physicians associated with medical colleges and having their private practice

Group IV - Post-graduate students pursuing Doctors of Medicine (MD)

A prior appointment was fixed with the physicians and they were approached personally by the investigator at a specified date and time. Questionnaires were distributed to individual physicians and were filled immediately in the presence of the investigator.

The questionnaire consisted of seven items, to collect information on physician's knowledge regarding relationship of oral health and systemic health, oral infection (periodontitis) as a potential risk factor for systemic complications (cardiovascular disease), whether the physician would tell the patient to maintain oral hygiene, referral for a dental opinion, and if they would advise medication if a patient approaches them with bleeding and/ or enlarged gums. They were also asked about their preference for referring their patients, whether to a dental specialist or general dental practitioner. Lastly, they were asked to report the source of their knowledge related to dentistry (literature/dentists/electronic media).

The data collected were analysed using SPSS version 11.5. Chi-square test and Fisher's exact test was applied to check the association between knowledge and practices.

\section{Results:}

The response rate for the survey was $52.3 \%$, as a total of 56 (out of the 106) physicians' practicing in Mangalore city completed the survey. The distribution of respondents was $21 / 27$ (82\%), 7/20(35\%) and 28/40(68\%) in Group I, Group III and Group IV respectively. Respondent's distribution based on gender and years of experience is shown in Table 1. About 19 physicians engaged only in private practice did not complete the questionnaire, so Group II was eliminated from the survey. Those who did not participate in the survey (especially those in Group II) cited lack of time, interest, or conflict with their busy practice schedule.

When considering periodontitis as a risk factor for Cardiovascular Disease it was seen that 24.9 percent of group IV (postgraduate students), but only 5.3 percent each of group I and III participants agreed it to be a major risk factor. 
If the patient asks physicians' opinion about bleeding/ enlargement of gingivae, $68.2 \%$ respondents from group I, all respondents of group III and $70.4 \%$ respondents from group IV stated that they would refer the patients for dental opinion. Though the entire respondent agreed that "oral health is related to systemic health", only $40 \%$ of the respondents ask about oral problems during general check- up. There was no statistical significance between groups on knowledge and practice when the chi-square test was applied. ( $p>0.05$ ). When the respondents were asked about the source of their knowledge related to dentistry, 12 stated literature (21.42\%), 6 stated the dentist (10.71\%), 36 stated literature and electronic media (64.28\%), and remaining 10 stated literature, electronic media and the dentist (17.85\%).

Table I. Physicians' distribution according to gender and years of experience

\begin{tabular}{llccc}
\hline & & Group I & Group III & Group IV \\
\hline Gender Distribution & Male & $19(87 \%)$ & $7(100 \%)$ & $24(89 \%)$ \\
& Female & $2(13 \%)$ & - & $4(11 \%)$ \\
Years of experience & $1-5$ years & $11(50 \%)$ & $2(29 \%)$ & - \\
& $6-10$ years & $8(36 \%)$ & - & - \\
\hline
\end{tabular}

Table II. Showing the responses of physicians' knowledge regarding "Oral health related to systemic health" and "Periodontal disease as risk factor for CVD"

\begin{tabular}{|c|c|c|c|c|c|}
\hline & $\begin{array}{c}\text { Overall } \\
\text { Response }\end{array}$ & Group I & Group III & Group IV & $\begin{array}{c}\text { Chi-square value, } \\
\text { df, p value }\end{array}$ \\
\hline Oral Health related to systemic health & $56(100 \%)$ & $21(100 \%)$ & $7(100 \%)$ & $28(100 \%)$ & - \\
\hline Major risk factor & $20(35.7 \%)$ & $3(5.3 \%)$ & $3(5.3 \%)$ & $14(24.9 \%)$ & $12.48,40.14^{*}$ \\
\hline Minor risk factor & $28(50 \%)$ & $14(25 \%)$ & $2(5.3 \%)$ & $12(21.4 \%)$ & \\
\hline Risk factor & $3(5.4 \%)$ & $1(1.8 \%)$ & $1(1.8 \%)$ & $1(1.8 \%)$ & \\
\hline Not a risk factor & $3(5.4 \%)$ & $2(3.6 \%)$ & - & $1(1.8 \%)$ & \\
\hline Do not know & $1(1.8 \%)$ & $1(1.8 \%)$ & - & - & - \\
\hline Not answered & $1(1.8 \%)$ & - & - & $1(1.8 \%)$ & - \\
\hline
\end{tabular}

*Statistically significant

Table III. Showing the responses of physicians' practices towards oral health of their patients

\begin{tabular}{|c|c|c|c|c|}
\hline & $\begin{array}{l}\text { Group I } \\
(\mathrm{N}=21)\end{array}$ & $\begin{array}{l}\text { Group III } \\
(\mathrm{N}=7)\end{array}$ & $\begin{array}{l}\text { Group IV } \\
(\mathrm{N}=28)\end{array}$ & $\begin{array}{c}\text { Chi-square value, } \\
\text { df,p value }\end{array}$ \\
\hline Ask about oral problems during check-up & $9(41 \%)$ & $3(43 \%)$ & $11(41 \%)$ & $3.04,2,0.21$ \\
\hline Refer to dentist in case of bleeding gums & $15(68 \%)$ & $7(100 \%)$ & $19(70 \%)$ & $8.25,10,0.60$ \\
\hline Refer to dental specialist & $8(53 \%)$ & $2(29 \%)$ & $6(33 \%)$ & $7.94,4,0.09$ \\
\hline Refer to periodontist & $2(25 \%)$ & $1(50 \%)$ & $3(50 \%)$ & $6.19,4,0.18$ \\
\hline Prescribe medicine & $14(64 \%)$ & $3(43 \%)$ & $8(30 \%)$ & $9.57,6,0.14$ \\
\hline
\end{tabular}




\section{Discussion:}

Certain systemic conditions may affect the initiation and progression of periodontal disease, most significantly being diabetes mellitus but evidence has also shed light on the converse side of the relationship between systemic health and oral health, that is, the potential effects of periodontal disease (PDD) on a wide range of organ systems especially the cardiovascular system. ${ }^{3,7,8}$ Although this information is easily available in medical and dental literature, little information is available regarding the knowledge of physicians' concerning the above facts.

Our survey included physicians who were Doctors of Medicine (MD), ranging from students to private practitioners and academicians. This population was selected because assessing and educating them would mean assessing/ educating the front-line doctors who would benefit from knowing the importance of 'oro-systemic link' and help in the prevention. Proper knowledge of oral diseases is crucial in medical practice due to the following reasons: (a) periodontal disease is associated with multiple systemic conditions of medical interest, (b) a large number of systemic diseases have oral manifestations, (c) many drugs are associated with oral adverse drug reactions, (d) physicians are generally not sufficiently trained to detect oral cancer manifestations, (e) physicians could play a pivotal role in oral public health, and (f) early detection of oral diseases by physicians could improve the oral health status of the population. ${ }^{9}$

Among physicians' practicing in Mangalore, this survey showed that knowledge regarding periodontal infection as a potential risk factor for systemic complications, the importance of regular dental check-ups, and the oral manifestations of periodontal disease was high amongst those in academic setting, both physicians as well as post graduate students. However, when it came into the incorporation of these in the medical practice, there was no statistical significance. The postgraduate students showed higher knowledge scores. This may be attributed to the fact that they remained in continuous touch with the recent advances and new facts coming to light on the topic. Continuing education programs may also have had some role to play in the difference in knowledge levels between the groups. A similar survey has been carried out among dental hygienists by Faden et al. in Saudi Arabia, where the awareness about the oral-systemic link was low. However, their results stated that $85 \%$ of their respondent's knowledge about periodontalsystemic interactions was current, and they provide comprehensive periodontal therapies to their patients. ${ }^{12}$ However, because this survey was evaluating the knowledge, attitude and practices among dental hygienists, who are generally trained to treat oral disease, it cannot be completely compared to our results here.

There are some limitations to this survey. The sample was confined to Mangalore city only. The physicians in sole private practice could not be surveyed as they refused participation either due to lack of time, interest, or conflict with their busy practice schedule. Due to the low response rate and the resulting sample size limiting to one city, it may not be possible to extrapolate the results of this survey to other settings in India or other parts of the world. However, it is important to assess the knowledge of physicians through larger studies similar to this, so as to make important changes in the medical school curriculum at grass root level. Also, additional training of physicians to recognize, initiate treatment, and refer to a dentist/periodontist, would be beneficial.

\section{Conclusion and Recommendations:}

The knowledge regarding the periodontal disease as a risk factor in CVD was high, however, the application of the knowledge in routine medical check-ups was observed to be less. A comparison of knowledge and practices between the groups showed no statistical significance. Because the dental disease affects almost all individuals, physicians should be able to recognize common conditions such as caries, periodontal disease, pulpitis, and dental abscess. In addition to initiating treatment and appropriate dental referrals, physicians should be familiar with the usage of antibiotics and medications in the perioperative period. Another important role for physicians is to help reduce the societal and economic impact of these diseases through patient education and prevention. The need for the physician to aid the dentist in preventive areas is to be stressed.

\section{References}

1. https://www.who.int/news-room/fact-sheets/detail/ cardiovascular-diseases-(cvds)

2. Parizi GN and Lori A. Periodontal Disease as a Risk Factor for Coronary Artery Disease American Journal of Applied Sciences 2005; 2:1526-1528.

3. Beck JD, Garcia RG, Heiss G, et al: Periodontal disease and cardiovascular disease. J Periodontol 1996; 67:1123

4. Liccardo D., Cannavo A., Spagnuolo G., Ferrara N., Cittadini A., Rengo C., Rengo G. Periodontal Disease: A Risk Factor for Diabetes and Cardiovascular Disease. Int. J. Mol. Sci. 2019;20:1414. doi: 10.3390/ijms20061414.

5. Wilder R, Robinson C, Jared H L, Boggess S L K. Obstetricians' knowledge and practice behaviors concerning periodontal health and preterm delivery and low birth weight. Journal of Dental Hygiene 2007; 81(4):81. 
6. Saini R, Saini S, Saini SR. Periodontal diseases: Arisk factor to cardiovascular disease. Ann Card Anaesth 2010;13:159

7. Hubbard T M. Periodontal disease and family physician. Am Fam Physician 1991;44:487-491

8. Nazir MA. Prevalence of periodontal disease, its association with systemic diseases and prevention. Int J Health Sci. 2017;11(2):72-80.

9. Ramirez JH, Arce R. Why must physicians know about oral diseases? Teach Learn Med 2010; 22:148-55.
10. Kirsch S, Coleton S. The role of the physician in the prevention of periodontal disease. Preventive Medicine. 1976; 5:347-359.

11. Genco G. Offenbacher G, Beck J. Periodontal disease and cardiovascular disease. J Am Dent Assoc 133;14S-22S.

12. Faden, Asma'a \& Alsalhani, Anas \& Idrees, Majdy \& Alshehri, Mohammed \& Nassani, Mohammad Zakaria \& Kujan, Omar. (2018). Knowledge, attitudes, and practice behavior of dental hygienists regarding the impact of systemic diseases on oral health. Saudi Medical Journal. 39. 1139-1147. 10.15537/smj.2018.11.23267. 